\title{
Perfil socioambiental de idosos de uma região do Pantanal Matogrossense: Um estudo das endoparasitoses
}

Socio-environmental profile of elderly people from a Matogrossense Pantanal region: A study of endoparasitises

Perfil socioambiental de personas mayores de una región Pantanal Matogrossense: Un estudio de endoparasitis

Tatiane Amorim de Matos ORCID: https://orcid.org/0000-0002-2898-1897 Universidade do Estado de Mato Grosso, Brasil

E-mail: tianeamatos@gmail.com.br

Andernice dos Santos Zanetti

ORCID: https://orcid.org/0000- 0001-7888-8183 Universidade do Estado de Mato Grosso, Brasil E-mail: andernice@gmail.com

Lucas França de Barros

ORCID: https://orcid.org/0000-0001-5203-178X Universidade do Estado de Mato Grosso, Brasil E-mail: lucasmariano.f@gmail.com

Marta dos Santos Miranda de Araújo ORCID: https://orcid.org/0000-0002-3165-8230 Hospital Regional de Cáceres Dr. Antônio Fontes, Brasil E-mail: martamiranda2@hotmail.com

Omar Ariel Espinosa ORCID: https://orcid.org/0000-0002-0912-9527 Faculdade do Pantanal, Brasil E-mail: omar.espinosa@fapan.edu.br

Antonio Francisco Malheiros

ORCID: https://orcid.org/0000-0001-8169-0557 Universidade do Estado de Mato Grosso, Brasil E-mail: malheiros@unemat.br

\begin{abstract}
Resumo
Como o processo natural do envelhecimento, o idoso apresenta mudanças morfofisiológicas e imunológicas deixando o mais susceptível as infecções parasitárias. As endoparasitoses constituem um problema de saúde pública no Brasil, apresentando variações de acordo com as condições de saneamento básico, nível socioeconômico, grau de escolaridade, idade e hábitos de higiene, entre outras variáveis. O objetivo geral deste trabalho foi analisar a prevalência de endoparasitas em idosos frequentadores do Centro de Convivência e idosos e funcionários da Instituição de Longa Permanência no município de Cáceres-MT. As amostras foram analisadas empregando três técnicas laboratoriais, sendo elas: Hoffmann, Ritchie e técnica de coloração Safranina modificado, no laboratório de Biologia Parasitaria da Universidade do Estado do Mato Grosso (UNEMAT). A prevalências de parasitas intestinais entre os idosos do Centro de Convivência (46,98\%), idosos da Instituição de Longa Permanência $(83,33 \%)$ e funcionários (84,61\%). Os endoparasitas Criptosporidium sp., Cyclospora sp. e Giardia lamblia foram os mais prevalentes, com $22 \%, 19 \%$ e $18 \%$, respectivamente. Esse estudo avaliou ainda as características sociodemográficas e hábitos de higiene pessoal dos idosos do Centro de Convivência e dos funcionários da ILP. Os resultados sugeriram uma condição epidemiológica preocupante da população idosa da ILP pois os altos índices de prevalência tanto nos idosos quanto nos funcionários evidenciam o alto risco de infecção e reinfecção nesse ambiente e mostra a necessidade de programas educacionais de conscientização das práticas higiênicas e de atenção básica a saúde da população idosa nessas instituições.
\end{abstract}

Palavra-chave: Geronte; Imunocomprometido; Saneamento básico; Ambiente.

\footnotetext{
Abstract

Like the natural aging process, the elderly person presents morphophysiological and immunological changes, making parasitic infections more susceptible. Endoparasitoses are a public health problem in Brazil, varying according to basic sanitation conditions, socioeconomic level, education level, age and hygiene habits, among other variables. The general objective of this work was to analyze the prevalence of endoparasites in elderly people who frequent the
} 
Centro de Convivência and the elderly and employees of the Long Term Care Institution in the municipality of Cáceres-MT. The samples were analyzed using three laboratory techniques, namely: Hoffmann, Ritchie and modified Safranina staining technique, in the Laboratory of Parasitic Biology at the State University of Mato Grosso (UNEMAT). THEprevalence of intestinal parasites among the elderly at the Community Center (46.98\%), the elderly at the Long Term Care Institution (83.33\%) and employees (84.61\%). The endoparasites Criptosporidium sp., Cyclospora sp. and Giardia lamblia were the most prevalent, with $22 \%, 19 \%$ and $18 \%$, respectively. This study also evaluated the sociodemographic characteristics and personal hygiene habits of the elderly at the Centro de Convivência and ILP employees. The results suggested a worrying epidemiological condition of the elderly population of the ILP because the high prevalence rates both in the elderly and in the employees show the high risk of infection and reinfection in this environment. and shows the need for educational programs for awareness of hygienic practices and primary health care for the elderly population in these institutions.

Keywords: Geronte; Immunocompromised; Basic sanitation; Environment.

\section{Resumen}

Al igual que el proceso de envejecimiento natural, el anciano presenta cambios morfofisiológicos e inmunológicos dejando a los más susceptibles a las infecciones parasitarias. La endoparasitosis es un problema de salud pública en Brasil, que varía según las condiciones de saneamiento básico, nivel socioeconómico, nivel educativo, edad y hábitos de higiene, entre otras variables. El objetivo general de este trabajo fue analizar la prevalencia de endoparásitos en ancianos que frecuentan el Centro de Convivência y ancianos y empleados de la Institución de Cuidados de Larga Duración en el municipio de Cáceres-MT. Las muestras se analizaron mediante tres técnicas de laboratorio, a saber: técnica de tinción de Hoffmann, Ritchie y Safranina modificada, en el Laboratorio de Biología Parasitaria de la Universidad Estatal de Mato Grosso (UNEMAT). LAprevalencia de parásitos intestinales entre ancianos del Centro Comunitario (46,98\%), ancianos de la Institución de Atención a Largo Plazo (83,33\%) y empleados $(84,61 \%)$. Los endoparásitos Criptosporidium sp., Cyclospora sp. y Giardia lamblia fueron las más prevalentes, con 22\%, 19\% y $18 \%$, respectivamente. Este estudio también evaluó las características sociodemográficas y los hábitos de higiene personal de los ancianos del Centro de Convivência y de los empleados del ILP. Los resultados sugirieron una condición epidemiológica preocupante de la población anciana del ILP porque las altas tasas de prevalencia tanto en ancianos como en el personal evidencian el alto riesgo de infección y reinfección en este entorno. y muestra el necesidad de programas educativos para conciencia de las prácticas higiénicas y atención primaria de salud para la población anciana en estas instituciones.

Palabras clave: Geronte; Inmunodeprimidos; Saneamiento básico; Medio ambiente.

\section{Introdução}

Os avanços tecnológicos e científicos associados ao maior controle sobre a saúde, meio ambiente, saneamento básico e melhorias no acesso aos serviços de saúde, ocorridos nas últimas décadas, contribuíram para o aumento da expectativa de vida e, consequentemente, com o envelhecimento populacional. (Freitas et al.,2016).

O aumento da expectativa de vida é um fenômeno natural, irreversível e mundial, diante desse processo de desenvolvimento, a inversão da pirâmide etária também é observada no Brasil. Segundo o Instituto Brasileiro de Geografia e Estatística (IBGE), o país tinha 21 milhões de pessoas com idade igual ou superior a 60 anos em 2012 e, com as melhorias na qualidade de vida, saúde e desenvolvimento, estima-se que em 2025 ocupará a sexta posição no mundo, em número de idosos, quando deve chegar a 32 milhões de pessoas com 60 anos ou mais. (IBGE, 2012; Freitas et al., 2016).

O envelhecimento, embora seja um processo natural, leva muitas vezes, a um quadro incapacitante, interferindo na autonomia e funcionalidade dos idosos, que evoluem com dificuldades para a realização de atividades cotidianas de forma independente, impactando expressivamente na qualidade de vida nessa faixa etária. (Freitas et al., 2016).

De acordo com Ely et al. (2011) as doenças infecciosas e/ou parasitárias em idosos ocorrem, principalmente, pelo envelhecimento do sistema imune e as manifestações vão desde obstrução intestinal, desnutrição, anemia, diarreia, má absorção, falta de apetite, náusea, vômito, dor abdominal que favorecem a flatulência e a constipação podendo resultar em comprometimento da capacidade funcional, da autonomia e qualidade de vida desse grupo etário. (Ely et al. 2011; Costa et al. 2014).

As parasitoses intestinais são frequentes em locais onde existem baixas condições socioeconômicas. (Justino et al., 2010). Os idosos residentes em Instituições de Longa Permanência (ILPs) apresentam maior grau de dependência e de doenças 
clínicas, em relação aos que vivem na comunidade, apresentando maior risco de adquirir doenças infecto parasitárias. (Ely et al., 2011).

Os parasitas intestinais necessitam de condições adequadas para que possam completar seu ciclo de vida, não dependendo apenas dos seus hospedeiros, mas sim de um ambiente propício, onde vários fatores irão interferir neste ciclo. (Saito, 2012).

Considerando o alto risco de endoparasitas em idosos, aumento da vulnerabilidade sobre o sistema imunológico devido à perda progressiva da autonomia dos idosos institucionalizados para o autocuidado que facilita os surtos e frequência de infecções de endoparasitoses, considerando a ausência de programas e estudos voltados para a população residente em ILPs.

Portanto o objetivo deste trabalho foi analisar a prevalência de endoparasitas em idosos frequentadores do Centro de Convivência e idosos e funcionários da Instituição de Longa Permanência no município de Cáceres-MT, correlacionando com variáveis socioambientais, econômicas e hábitos de higiene.

\section{Metodologia}

\subsection{Tipo, Área e População de estudo}

Trata-se de um estudo descritivo, quantitativo e transversal com base em fontes primárias e amostras não probabilísticas. Estudos transversais ou de corte transversal aqueles que produzem "instantâneos" da situação de saúde de uma população ou comunidade com base na avaliação individual do estado de saúde de cada um dos membros do grupo, e também determinar indicadores globais de saúde para o grupo investigado. (Rouquayrol \& Filho,2003).

O estudo foi desenvolvido no município de Cáceres situado no sudoeste de Mato Grosso, região centro-oeste do Brasil, integrando a microrregião do alto Pantanal e a mesorregião do centro-sul mato-grossense, com uma área territorial de $24.796,8 \mathrm{~km}^{2}$ e se encontra situada a $215 \mathrm{~km}$ da capital, Cuiabá, é o sexto município com maior população estimada do estado, 94. 861 habitantes e uma população com 60 anos ou mais é de 9,4\% da população. (IBGE,2020).

A população estudada é formada por idosos de ambos os sexos com idade mínima de 60 anos que reside nas Instituições de longa permanência Lar Serva de Maria e idosos que frequentam o Centro de convivência Remanso Fraterno; também foram incluídos na pesquisa os enfermeiros, técnicos de enfermagem, cuidadores e manipuladores de alimentos que trabalham nestas instituições.

O Lar Serva de Maria é uma Instituição de Longa Permanência, fundada em 14 de maio de 1972 no município de Cáceres. É uma instituição filantrópica, tendo sua diretoria formada por membros do Centro Espirita Matheus. Abriga hoje, 36 internos, sendo 19 homens e 17 mulheres e possui 15 funcionários, uma assistente social, quatro técnicos de enfermagem, quatro cuidadores, duas faxineiras, uma cozinheira, uma lavadeira e um motorista.

O Centro de convivência Remanso Fraterno João Gabriel é uma instituição de convivência transitória de idosos que foi fundada em 05 de março de 2004. É uma instituição filantrópica e seus recursos financeiros vêm das mensalidades dos membros da diretoria e doações. A instituição não tem enfermeiro, técnico de enfermagem e nem cuidadores fixos. Todos os trabalhadores são voluntários aleatórios.

\subsection{Critério de inclusão e exclusão}

Como critério de inclusão: Idosos residentes na Instituição de longa permanência; idosos que frequentam o Centro de convivência Remanso Fraterno; funcionários que trabalham nestas instituições do Município de Cáceres.

Critérios de exclusão: Idoso e funcionários que não aceitaram participar e não assinaram o Termo de Consentimento. 


\subsection{Coletas de dados}

Os idosos e os funcionários foram convidados a participar da pesquisa, após leitura e assinatura do Termo de Consentimento Livre e Esclarecido (TCLE). Um inquérito epidemiológico com dados de perfil demográfico, ambiental e de higiene pessoal foi aplicado a cada participante por meio de fichas de questionário padronizado.

Um segundo questionário foi aplicado às Instituições, abordando a obtenção de alimentos, procedência da água, rede de esgoto, treinamento de pessoal e número de pessoas envolvidas na funcionalidade da instituição.

Para a composição de banco de dados das amostras fecais, foram entregues frascos coletores descartáveis e estéril para cada participante, devidamente identificados e posteriormente o material coletado foi encaminhado ao Laboratório de Biologia Parasitária - LaBPar da Universidade do Estado de Mato Grosso - UNEMAT, situado em Cáceres - MT, para análises coprológicas.

As amostras foram analisadas empregando duas técnicas laboratoriais, sendo elas: sedimentação espontânea Hoffmann e a técnica de sedimentação por centrifugação - Ritchie e uma técnica de coloração - Método Safranina modificado.

\subsection{Análise dos dados}

Os dados foram compilados em Excel versão 2010 para análise estatística. Para análise descritiva das variáveis continuas foi utilizada medidas de tendência central e para variáveis categóricas usamos a frequência e o teste de Qui-quadrado de Person.

Para análise de associação foi utilizado o método de Odds rattios (OR) que é definida como a razão entre a chance de um evento ocorrer em um grupo e a chance de ocorrer em outro grupo, mostrando qual grupo tem maior risco de estar parasitado. Foram considerados estatisticamente significantes os valores de $p$-valor $\leq 0,005$.

\subsection{Aspectos éticos}

Todas as etapas do trabalho seguiram os princípios éticos e morais. Os aspectos éticos em atividades da pesquisa que envolva seres humanos são regulados pelas Diretrizes e Normas de Pesquisa em Seres Humanos, através da Resolução 196/96 do Conselho Nacional de Saúde. O Termo de Consentimento Livre e Esclarecido, Número do parecer foi devidamente avaliado pelo Comitê de Ética de Pesquisa - CEP da Universidade do Estado de Mato Grosso, sob o n. 3.572.397.

\section{Resultados}

Neste estudo foram coletadas 119 amostras fecais de idosos das duas Instituições no município de Cáceres - MT. Sendo 30,25\% (36) das amostras dos Internos da Instituição de Longa Permanência (ILP) Lar Serva de Maria e 69,75\% (83) do Centro de convivência Remanso Fraterno.

Dos 15 funcionários da ILP, 13 (87\%) participaram deste estudo. O Centro de convivência Remanso Fraterno não possui funcionários fixos, o trabalho é de caráter voluntário, portanto não participaram dessa pesquisa.

\subsection{Prevalência endoparasitária na população geronte estudada}

Dos 119 resultados parasitológicos analisados pelas técnicas de Hoffman, de Ritchie e o método de coloração de Safranina modificada, a taxa de positividade geral foi de 57,98\% (69). Os endoparasitas mais prevalentes foram: Cryptosporidium sp. 39,13\% (27), Giardia lamblia 36,23\% (25), Cyclospora sp. 31,88\% (22), Entamoeba coli 28,98\% (20) Endolimax nana 20,28\% (14) e Blastocystis hominis 18,84\% (13). Os parasitas com menor frequência Ancilostomídeo 8,69\% (6), Entamoeba histolytica/dispar 2,89\% (2), Enterobius vermicularis 1,44\% (1) e Sarcocystis sp. 1,44\% (1) (Figura 1). 
Foram encontrados endoparasitas de dez espécies diferentes, oito no grupo dos protozoários e dois no grupo dos helmintos. Os protozoários de maior ocorrência foram Cryptosporidium sp. com 53,33\% (16) no Lar Serva de Maria em comparação com 28,20\% (11) no Remanso Fraterno, Giardia lamblia com 50\% (15) no Lar Serva de Maria em comparação com 25,64\% (10) no Remanso Fraterno e Cyclospora sp. com 46,66\% (14) no Lar Serva de Maria em comparação com 20,51\% (8) no Remanso Fraterno. Os helmintos foram Ancylostomídeo com 6,66\% (2) no Lar Serva de Maria em comparação com 10,25\% (4) no Remanso Fraterno e Enterobius vermicularis com 2,56\% (1) no Remanso Fraterno em comparação com nenhum caso no Lar Serva de Maria (Figura 1).

Figura 1. Comparação da prevalência de espécies endoparasitárias entre os idosos das Instituições analisadas no município de Cáceres-MT, 2020.

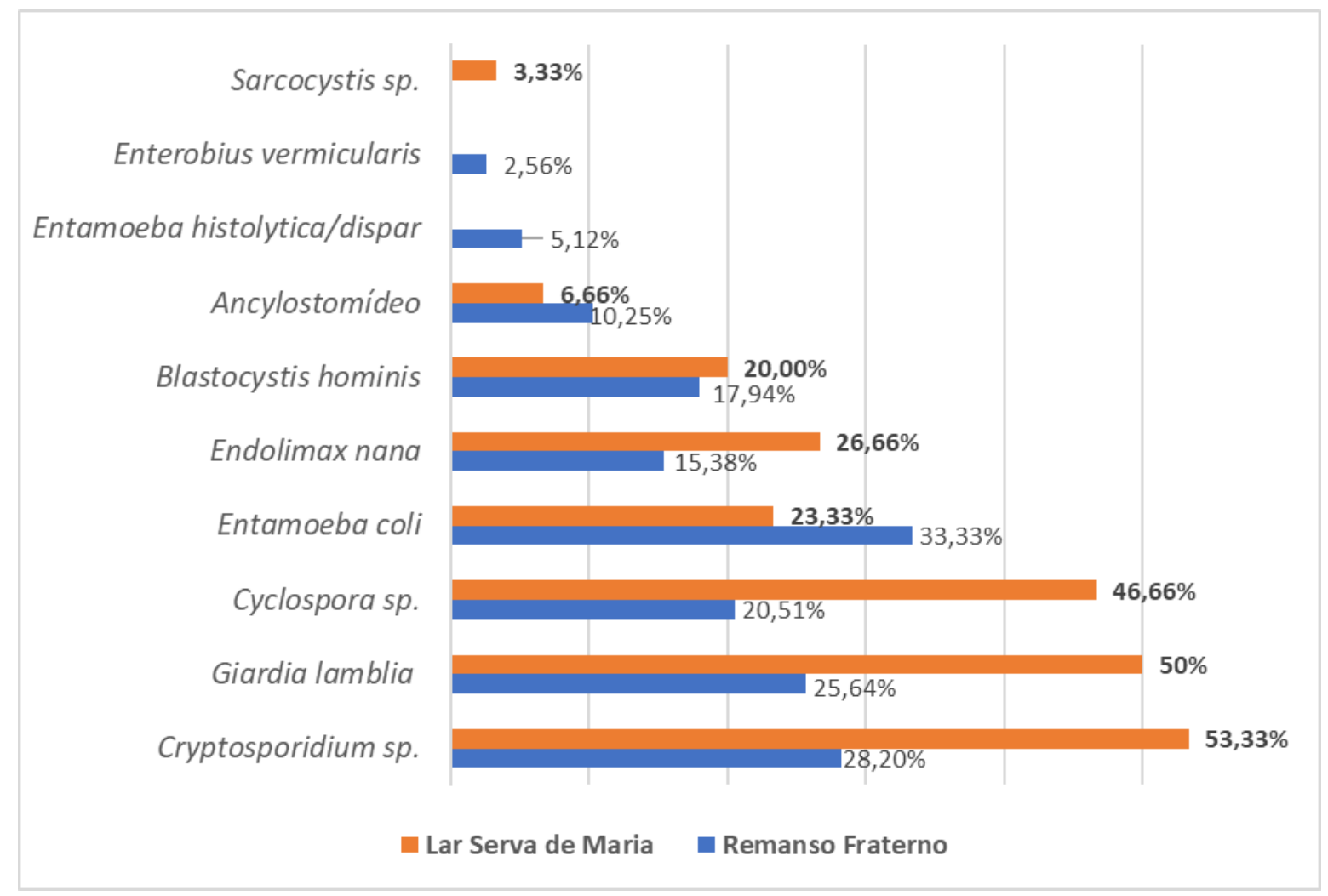

Fonte: Autores.

O perfil da população geronte analisada foi categorizado segundo o sexo, idade e local de domicílio. Foram examinadas 43 amostras do sexo masculino com positividade de $36,24 \%$, e 76 do sexo feminino com positividade de $63,76 \%$. Quanto à prevalência por grupo etário, verificou-se que os idosos de 60 a 70 anos (77) foram os mais parasitados com 66,66\%, seguidos de idosos de 71 a 80 anos (37) com 31,89\% e idosos maiores de 80 anos (5) com 1,45\%.

Os resultados apontaram a presença de poliparasitismo, com uma prevalência geral da população geronte analisada de $55,07 \%$ (38) das amostras positivas. Nas amostras do ILP o poliparasitismo foi encontrado em 76,66\% (23) das amostras positivas e no Centro de convivência 38,46\% (15). O monoparasitismo foi encontrado em 44,92\% (31) das amostras gerais positivas da população geronte. Nas amostras do ILP o monoparasitismo foi encontrado em 23,33\% (7) das amostras positivas e no Centro de convivência $61,53 \%$ (24).

Nas duas instituições, ILP e Centro de convivência, a associação dupla de espécies foi a mais observada, 47,82\% e 61,57\% respectivamente. No Centro de convivência observou-se maior percentual na associação de Cryptosporidium sp. + 
Cyclospora sp. (18,45\%) e na ILP Serva de Maria observou-se maior percentual na associação das espécies Giardia lamblia + Cryptosporidium sp. + Cyclospora sp. (17,40\%).

\subsection{Prevalência endoparasitária nos funcionários da instituição de longa permanência}

Dos 15 funcionário da ILP somente 13 participaram deste estudo. Do total das amostras coletadas dos funcionários, observou-se uma positividade de 84,61\% (11) para algum parasita intestinal.

Dentre os parasitas intestinais diagnosticados nos servidores, os de maior prevalência foram: Cyclospora sp. (72,72\%), Cryptosporidium sp. (63,63\%), Giardia lamblia (27,27\%) e com menor frequência Entamoeba coli (18,18\%), Endolimax nana (9,09\%) e Sarcocystis sp. (9,09\%).

Os resultados das amostras parasitológicas dos funcionários da ILP Lar Serva de Maria apontaram a presença de poliparasitismo, com uma prevalência de 69,23\% (9) amostras positivas com mais de um endoparasito. O monoparasitismo foi encontrado em $15,38 \%$ (2) das amostras positivas.

A associação dupla de espécies entre os funcionários foi a mais observada, em 53,84\% (7). A associação de Cryptosporidium sp. + Cyclospora sp. foi a mais prevalente em 30,77\% (4). As associações duplas entre Cryptosporidium sp. + Sarcocystis sp.; Entamoeba coli + Cryptosporidium sp. e Endolimax nana + Giardia lamblia, apresentaram cada uma 7,69\% de prevalência. Infecções triplas e quádruplas estavam presentes em apenas uma amostra de cada.

\subsection{Comparação das prevalências de endoparasitas em idosos das duas instituições e funcionários da ILP}

A prevalência de parasitas intestinais encontrada nos idosos do Centro de Convivência foi de 46,98\% e nos idosos da ILP foi de 83,33\%. Já nos funcionários da ILP a prevalência endoparasitária foi de 84,61\% (Tabela 1).

Tabela 1. Prevalências de endoparasitas nas amostras de idosos e funcionários da Instituição de Longa Permanência (ILP) Lar Servas de Maria e idosos do Centro de Convivência Remanso Fraterno, na cidade de Cáceres, MT, 2020.

\begin{tabular}{lcc}
\multicolumn{1}{c}{ População } & N & \% \\
\hline Idosos do Centro de Convivência & & \\
Amostras Negativas & 44 & 53,02 \\
Amostras Positivas & 39 & 46,98 \\
Idosos da Instituição de Longa Permanência & & \\
Amostras Negativas & 6 & 16,67 \\
Amostras Positivas & 30 & 83,33 \\
Funcionários da Instituição de Longa Permanência & & \\
Amostras Negativas & 2 & 15,39 \\
Amostras Positivas & 11 & 84,61 \\
\hline
\end{tabular}

Fonte: Autores.

Na prevalência geral combinada dos endoparasitas nas amostras dos idosos das duas instituições e dos funcionários da ILP, observamos que os endoparasitas de maiores prevalências foram: Cryptosporidium sp. (22\%), Cyclospora sp. (19\%), Giardia lamblia (18\%) e menos prevalentes: Sarcocystis sp. (1\%), Strongyloides stercoralis (1\%) e Enterobius vermicularis (1\%) (Tabela 2). 
Comparando os resultados das análises nas amostras dos idosos das duas instituições e dos funcionários da ILP Lar serva de Maria, é possível observar que os protozoários mais prevalentes nas amostras totais, Cryptosporidium sp. e Cyclospora sp., apresentaram maiores prevalências entre os funcionários e idosos da ILP (Tabela 2).

Giardia lamblia apresentou prevalências similares entre os idosos das duas instituições e os funcionários da ILP. Entamoeba coli foi mais prevalente nos idosos da Instituição transitória Remanso Fraterno e E. histolytica/dispar foi identificada apenas nos idosos dessa instituição (Tabela 2).

Os helmintos identificados nas amostras, Ancilostomídeos, Enterobius vermiculares e Strongyloides stercoralis, apresentaram baixas prevalências nos três grupos analisados (Tabela 2).

Tabela 2. Prevalência das espécies de endoparasitas encontrados em idosos e funcionários da Instituição de Longa Permanência (ILP) Lar Servas de Maria e idosos do Centro de Convivência Remanso Fraterno, na cidade de Cáceres, MT, 2020.

\begin{tabular}{|c|c|c|c|c|}
\hline Parasitas intestinais & $\begin{array}{l}\text { Idosos da ILP Lar } \\
\text { Servas de Maria N } \\
\text { - \% (IC 95\%) }\end{array}$ & $\begin{array}{l}\text { Funcionários da } \\
\text { ILP Lar Servas } \\
\text { de Maria N - \% } \\
\text { (IC 95\%) }\end{array}$ & $\begin{array}{c}\text { Instituição Remanso } \\
\text { Fraterno N - \% (IC } \\
95 \%)\end{array}$ & $\begin{array}{c}\text { Total N - \% (IC } \\
95 \%)\end{array}$ \\
\hline \multicolumn{5}{|l|}{ PROTOZOÁRIOS } \\
\hline Cryptosporidium sp. & $16-23 \%(15-34)$ & $7-32 \%(16-53)$ & $11-18 \%(10-29)$ & $34-22 \%(17-27)$ \\
\hline Cyclospora sp. & $14-20 \%(12-31)$ & $8-36 \%(20-57)$ & $8-13 \%(7-23)$ & $30-19 \%(13-25)$ \\
\hline Giardia lamblia & $15-22 \%(14-33)$ & $3-14 \%(5-33)$ & $10-16 \%(9-27)$ & $28-18 \%(14-22)$ \\
\hline Entamoeba coli & $7-10 \%(5-19)$ & $2-9 \%(3-28)$ & $13-21 \%(13-33)$ & $22-14 \%(9-18)$ \\
\hline Endolimax nana & $8-12 \%(6-21)$ & $1-5 \%(1-22)$ & $6-10 \%(5-20)$ & $15-9 \%(6-13)$ \\
\hline Blastocystis hominis & $6-9 \%(4-18)$ & 0 & $7-11 \%(6-22)$ & $13-9 \%(6-12)$ \\
\hline E. histolytica/dispar* & 0 & 0 & $2-3 \%(1-11)$ & $2-2 \%(0-3)$ \\
\hline Sarcocystis sp. & $1-1 \%(0-8)$ & 0 & 0 & $1-1 \%(0-2)$ \\
\hline \multicolumn{5}{|l|}{ HELMINTOS } \\
\hline Ancilostomídeos & $2-3 \%(1-10)$ & 0 & $4-6 \%(3-15)$ & $6-4 \%(2-6)$ \\
\hline Enterobius vermicularis & 0 & 0 & $1-2 \%(0-9)$ & $1-1 \%(0-2)$ \\
\hline Strongyloides stercoralis & 0 & $1-5 \%(1-22)$ & 0 & $1-1 \%(1-2)$ \\
\hline
\end{tabular}

* O diagnóstico laboratorial diferencial entre Entamoeba histolytica e Entamoeba dispar não pode ser realizado tomando-se como base a morfologia, a não ser que sejam vistas hemácias ingeridas pelos trofozoítos (E. histolytica).

Fonte: Autores.

\subsection{Perfil ambiental das duas instituição de atendimento ao idoso}

Um questionário foi aplicado nas duas instituições estudadas e respondido pelos coordenadores responsáveis. De acordo com os resultados da avaliação ambiental, os itens que mais se destacaram foram referentes à estrutura das instituições que são de alvenaria e as dependências externas são de terra e cimento. Há água encanada e de poço artesiano para consumo e para rega da horta. A purificação da água antes do consumo é feita pelo filtro. Os escoadores de esgoto são por fossas. Outros aspectos avaliados foram: como é o destino do lixo doméstico, que é coletado 3 vezes por semana; a roupa de cama e banho da ILP Lar serva de Maria é higienizada com água, sabão e água sanitária e a troca das roupas de cama e toalha é realizada diariamente (Tabela 3). 
As Instituições abrigam animais doméstico como cães, gatos e aves, com contato direto com os idosos. Os animais não são vermifugados. As duas instituições possuem hortas que utilizam esterco animal e adubo químico para o cultivo de alface, couve, cebolinha dentre outras hortaliças, que são consumidas pelos idosos (Tabela 3).

Os coordenadores relataram que os funcionários não possuem treinamento em relação a higiene pessoal e o uso dos equipamentos de proteção individual.

Tabela 3. Dados ambientais e de higiene das Instituição de Longa Permanência Lar Serva de Maria e do Centro de Convivência Remanso Fraterno, na cidade de Cáceres, MT, 2020.

\begin{tabular}{|c|c|c|}
\hline & $\begin{array}{c}\text { Instituição Lar Serva de } \\
\text { Maria }\end{array}$ & $\begin{array}{c}\text { Instituição } \\
\text { Remanso Fraterno } \\
\end{array}$ \\
\hline \multicolumn{3}{|l|}{$\begin{array}{r}\text { Características } \\
\end{array}$} \\
\hline Estrutura Material & Alvenaria & Alvenaria \\
\hline Tipo de piso & Cerâmica/ cimento & Cerâmica/ cimento \\
\hline Quintal & Terra / cimento & Terra / cimento \\
\hline Rua & Asfalto & Chão batido \\
\hline Escoamento de esgoto & Fossa & Fossa \\
\hline \multirow[t]{2}{*}{ Água encanada } & Sim & Sim \\
\hline & Poço artesiano & Poço artesiano \\
\hline Tratamento da água consumida & Filtrada & Filtrada \\
\hline Coleta de lixo & Sim / 3 x semana & Sim / 3 x semana \\
\hline Possui Horta & Sim & Sim \\
\hline Utilização de adubo & Adubo animal & Adubo animal / químico \\
\hline Hortaliças produzidas & Couve / rúcula /cebolinha & $\begin{array}{c}\text { Alface / couve / rúcula / } \\
\text { cenoura / outros }\end{array}$ \\
\hline Hortaliças para consumo próprio & Sim & Sim \\
\hline \multirow[t]{2}{*}{ Possui animais domestico } & Sim & Sim \\
\hline & Gatos / aves & Cão / aves \\
\hline Animais vermifugados & Não & Não \\
\hline Idoso tem contato com os animais & Sim & Sim \\
\hline Quantos banheiros & 8 & 5 \\
\hline Lavagem do banheiro & Diariamente & 2x semana \\
\hline Produtos para lavagem dos banheiros & $\begin{array}{c}\text { Sabão / água sanitária / } \\
\text { desinfetante }\end{array}$ & $\begin{array}{c}\text { Sabão / água sanitária / } \\
\text { desinfetante }\end{array}$ \\
\hline Lavagem das roupas / roupa de cama & Água / sabão / água sanitária & $*$ \\
\hline Troca de roupa de cama e toalhas & Diariamente & $*$ \\
\hline Os idosos dividem a mesma toalha & Não & $*$ \\
\hline Número de idosos por quarto & 2 & $*$ \\
\hline Lavagem da cozinha & Diariamente & Sempre que usada \\
\hline Refeição preparada na Instituição & Sim & Sim \\
\hline Tiveram palestras de educação em saúde & Não & Não \\
\hline
\end{tabular}

*Não se aplica por não ser uma Instituição de Longa Permanência.

Fonte: Autores.

\subsection{Perfil sócio demográfico e ambiental dos idosos do centro de convivência}

O perfil socioeconômico e demográfico dos idosos do ILP não foram levantados nessa pesquisa, pois a maioria dos internos estão em estado de demência, impossibilitando a aplicação de questionário.

O perfil socioeconômico e demográfico dos idosos atendidos no Centro de Convivência, permitiu identificar fatores relativos à saúde e higiene, relações sociais e ambientais existentes nas residências dos mesmos (Tabela 4).

De acordo com os resultados da avaliação socioeconômica e demográfica, os itens que mais se destacaram foram referentes à predominância do sexo feminino com $73,49 \%$ e masculino com $26,51 \%$. A prevalência de parasitas intestinais em 
ambos sexos foram similares. A faixa de renda dos entrevistados, variou de um ou dois salários mínimos, considerando o valor de $\mathrm{R} \$ 1.045,00$, vigente na época da coleta de dados. A prevalência de endoparasitas foi maior nas faixas de renda de dois a três salários mínimos. Em relação a cor, 50,60\% dos entrevistados se declararam pardo, 15,67\% negros, 6,02\% brancos e $1,20 \%$ indígena. Dos entrevistados 26,51\% não se declararam quanto a cor. Brancos e negros apresentaram maior prevalência de parasitas intestinais (Tabela 4).

Outros aspectos avaliados, relacionados à saúde dos idosos, podem ser observados. Destaca-se, nesse contexto, o alto índice de idosos com hipertensão $(24,09 \%)$, diabetes $(14,45 \%)$ e hipertensão e diabetes $(14,45 \%)$, todos apresentando altas prevalências parasitárias (Tabela 4).

Referente às características ambientais dos pacientes, $84,34 \%$ dos participantes referiram ter casa de alvenaria e 15,66\% a casa é de madeira. Quanto ao tipo de piso da casa, $95,19 \%$ possuem piso do tipo cerâmica; 50,57\% o quintal é de terra e 40,96\% o tratamento de água antes do consumo e feito pelo filtro de barro. Os escoadores de esgoto são feitos por fossa em 79,52\% e apena 15,66\% possui rede de esgoto. Dos entrevistados, 6,02\% queimam o seu lixo doméstico. Observando essas variáveis, todas apresentaram valores similares na prevalência de parasitas intestinais (Tabela 4).

Quando observado os hábitos de higiene, 84,34\% referem sempre lavar as mãos após o uso do banheiro, 71,08\% sempre lavam as mãos antes de alimentar-se, $15,66 \%$ preferem andar descalço em casa, 34,94\% referem utilizar apenas água para lavar frutas e verduras para consumo e 65,06\% fervem o leite antes do consumo. Essas variáveis também apresentaram valores similares na prevalência de parasitas intestinais (Tabela 4).

A Tabela 4 apresenta em detalhes a prevalência de parasitas intestinais em cada variável analisada e o respectivo valor de $p$.

Tabela 4. Dados sociodemográficos, ambientais, de saúde e de higiene dos idosos da Instituição Remanso Fraterno e a prevalência de parasitas intestinais em cada variável.

\begin{tabular}{lccc}
\hline Características sociodemográficas & $\begin{array}{c}\text { População } \\
\text { analisada } \\
\mathrm{N}(\%)\end{array}$ & $\begin{array}{c}\text { Prevalência de } \\
\text { parasitas intestinais } \\
\mathrm{N}(\%)\end{array}$ & $P^{*}$ \\
\hline Sexo & & & \\
Feminino & $61(73,49)$ & $29(47,54)$ & 0,8665 \\
Masculino & $22(26,51)$ & $10(45,45)$ & \\
Idade & & & \\
$60-70$ & $62(74,70)$ & $28(45,16)$ & \\
$71-80$ & $19(22,89)$ & $9(47,37)$ & 0,3167 \\
$81-90$ & $2(2,41)$ & $2(100)$ & \\
Cor & & & \\
Pardo & $42(50,60)$ & $23(54,76)$ & \\
Branco & $5(6,02)$ & $4(80,00)$ & 0,1027 \\
Negro & $13(15,67)$ & $11(84,61)$ & \\
Indígena & $1(1,20)$ & $0(0)$ & \\
Não declarou & $22(26,51)$ & $1(4,55)$ & \\
Escolaridade & & & \\
Analfabeto & $24(28,91)$ & $9(37,50)$ & \\
Fundamental incompleto & $38(45,79)$ & $16(42,11)$ & 0,1703 \\
Fundamental completo & $7(8,43)$ & $5(71,43)$ & \\
Médio incompleto & $1(1,20)$ & $0(0)$ & \\
Médio completo & $11(13,26)$ & $7(63,64)$ & \\
Superior incompleto & $2(2,41)$ & $2(100)$ & \\
& & &
\end{tabular}




\begin{tabular}{|c|c|c|c|}
\hline \multicolumn{4}{|l|}{ Renda } \\
\hline Até 1 salário & $40(48,19)$ & $16(40,00)$ & \multirow{4}{*}{0,3114} \\
\hline 1-2 salários & $28(33,74)$ & $15(53,57)$ & \\
\hline 2-3 salários & $8(9,64)$ & $5(62,50)$ & \\
\hline 3-4 salários & $7(8,43)$ & $3(42,85)$ & \\
\hline \multicolumn{4}{|c|}{ Características ambientais } \\
\hline \multicolumn{4}{|c|}{ Moradia } \\
\hline Própria & $69(83,13)$ & $33(47,83)$ & \multirow{3}{*}{0,6779} \\
\hline Alugada & $10(12,05)$ & $5(50,00)$ & \\
\hline Cedida & $4(4,82)$ & $1(25,00)$ & \\
\hline \multicolumn{4}{|l|}{ Material da casa } \\
\hline Alvenaria & $70(84,34)$ & $34(48,57)$ & \multirow[t]{2}{*}{0,5639} \\
\hline Madeira & $13(15,66)$ & $5(38,46)$ & \\
\hline \multicolumn{4}{|l|}{ Tipo de piso } \\
\hline Chão batido & $3(3,61)$ & $3(100)$ & \multirow{3}{*}{0,0830} \\
\hline Cerâmica & $79(95,19)$ & $35(44,30)$ & \\
\hline Cimento & $1(1,20)$ & $1(100)$ & \\
\hline \multicolumn{4}{|l|}{ Quintal } \\
\hline Terra & $42(50,57)$ & $21(50,00)$ & \multirow{6}{*}{0,3180} \\
\hline Grama & $4(4,82)$ & $1(25,00)$ & \\
\hline Cimento & $12(14,47)$ & $4(33,33)$ & \\
\hline Piso/cerâmica & $3(3,61)$ & $2(66,67)$ & \\
\hline Terra e cimento & $16(19,29)$ & $10(62,50)$ & \\
\hline Terra e grama & $6(7,24)$ & $1(16,67)$ & \\
\hline \multicolumn{4}{|c|}{ Possui animal de estimação } \\
\hline Sim & $68(81,93)$ & $32(47,06)$ & \multirow[b]{2}{*}{0,9395} \\
\hline Não & $15(18,07)$ & $7(46,67)$ & \\
\hline \multicolumn{4}{|l|}{ Rua } \\
\hline Asfalto & $60(72,29)$ & $28(46,67)$ & \multirow{4}{*}{0,0100} \\
\hline Chão batido & $14(16,87)$ & $11(78,57)$ & \\
\hline Paralelepípedo & $1(1,20)$ & $0(0)$ & \\
\hline Não responderam & $8(9,64)$ & $0(0)$ & \\
\hline \multicolumn{4}{|l|}{ Escoamento de esgoto } \\
\hline Rede geral & $13(15,66)$ & $4(30,77)$ & \multirow[t]{3}{*}{0,2734} \\
\hline Fossa & $66(79,52)$ & $32(48,48)$ & \\
\hline Não sei & $4(4,82)$ & $3(75,00)$ & \\
\hline \multicolumn{4}{|l|}{ Coleta de lixo } \\
\hline $2 \mathrm{X}$ & $6(7,23)$ & $3(50,00)$ & \multirow[t]{3}{*}{0,6763} \\
\hline $3 \mathrm{X}$ & $68(81,93)$ & $32(57,06)$ & \\
\hline Não sei & $9(10,84)$ & $4(44,44)$ & \\
\hline \multicolumn{4}{|l|}{ Destino do lixo } \\
\hline Prefeitura - carro & $77(92,78)$ & $35(45,45)$ & \multirow{3}{*}{0,1935} \\
\hline Enterra & $1(1,20)$ & $0(0)$ & \\
\hline Queima & $5(6,02)$ & $4(80,00)$ & \\
\hline \multicolumn{4}{|c|}{ Saúde e hábitos de higiene } \\
\hline Doença & & & \\
\hline Diabete & $12(14,46)$ & $5(41,67)$ & \\
\hline Hipertensão & $20(24,10)$ & $14(70,00)$ & \\
\hline Hipertensão e diabete & $12(14,46)$ & $5(41,67)$ & 0,2102 \\
\hline Outras & $30(36,14)$ & $12(40,00)$ & \\
\hline Nenhuma & $9(10,84)$ & $3(33,33)$ & \\
\hline
\end{tabular}


Quando realizou exames de fezes

Ano passado

2 anos

3 anos

Faz tempo

Não lembro

Tratamento de água

Filtro de barro

Fervura

Mineral

Filtro com vela

Torneira

Outro

Banho ao dia

$1 \mathrm{X}$

$2 \mathrm{X}$

$3 \mathrm{X}$

4X

Mais

Costuma andar descalço

Sim

Não

As vezes

Limpeza da casa

Diariamente

4X na semana

$3 \mathrm{X}$ na semana

2X na semana

Lavagem das mãos após banheiro

Sempre

Às vezes

Nunca

Lavagem das mãos após contato com animais

Sempre

Às vezes

Nunca

Lavagem das mãos antes de alimentar-

se

Sempre

Às vezes

Nunca

Tratamento das frutas e verduras

Com água

Água e sabão

Água sanitária

Vinagre

Outros

Tratamento do leite

Nenhum

Fervura

Outro
$31(37,36)$

$13(15,66)$

$3(3,61)$

$21(25,30)$

$15(18,07)$

$34(40,96)$

$4(4,82)$

$27(32,54)$

$9(10,84)$

$2(2,41)$

$7(8,43)$

$5(6,02)$

$52(62,66)$

$14(16,87)$

$11(13,25)$

$1(1,20)$

$13(15,66)$

$55(66,27)$

$15(18,07)$

$55(66,27)$

$4(4,82)$

$11(13,25)$

$13(15,66)$

$70(84,34)$

$10(12,05)$

$3(3,61)$

$66(79,52)$

$14(16,87)$

$3(3,61)$

$59(71,08)$

$20(24,10)$

$4(4,82)$

$28(47,46)$

$29(34,94)$

$20(24,10)$

$17(20,48)$

$13(15,66)$

$4(4,82)$

$21(25,30)$

$54(65,06)$

$8(9,64)$
$33(50,00)$

$4(28,57)$

$2(66,67)$

$9(45,00)$

$2(50,00)$

0,2609

0,5871

0,2449

0,9068

0,3498

$5(45,45)$

$4(30,77)$

$35(50,00)$

$2(20,00)$

0,0723

$2(66,67)$

0,1677

0,9833

$14(48,28)$

$8(40,00)$

$10(58,82)$

$5(38,46)$

$2(50,00)$

$12(57,14)$

$25(46,29)$

$2(25,00)$

* Valor de $P$ calculado pelo teste de Pearson do Qui-quadrado. Fonte: Autores. 


\subsection{Perfil sócio demográfico e ambiental dos funcionários da instituição de longa permanência}

Foi observado que a maioria deles é do sexo feminino $76,92 \%$. Cerca de $46,15 \%$ possuíam renda entre um e dois salários mínimos. Quanto à escolaridade, 61,55\% possuíam o ensino médio completo. Das residências, 92,30\% são de alvenaria e nas casas de todos esses profissionais, há água encanada. Grande parte deles, 53,84\% afirmaram consumir água do filtro de barro (Tabela 5).

A situação sanitária mostra que o escoamento do esgoto é feita por fossa em 76,92\% da residência dos funcionários. Quando observado os hábitos de higiene, a maioria desses profissionais, 76,92\%, afirmaram lavar as mãos após o uso do banheiro e lavarem as mãos antes de alimentar-se. No aspecto de cuidados com os alimentos, 61,54\% referem utilizar apenas água para lavar frutas e verduras para o consumo. Dos funcionários entrevistados, 76,92\% (10) mencionaram já terem feito exame de fezes, mas a maioria deles $61,53 \%$ (8) fizeram exames há mais de um ano (Tabela 5).

Todas as variáveis do perfil dos funcionários analisadas, apresentaram altos valores de prevalência de parasitas intestinais. A tabela 5 apresenta em detalhes a prevalência dos endoparasitas em cada variável analisadas.

Tabela 5. Dados sociodemográficos, ambientais, de saúde e de higiene dos funcionários da Instituição de Longa Permanência Lar Servas de Maria e as prevalências de parasitas intestinais em cada variável.

\begin{tabular}{|c|c|c|}
\hline & $\begin{array}{l}\text { População analisada } \\
\text { N }(\%)\end{array}$ & $\begin{array}{l}\text { Prevalência de parasitas intestinais } \\
\qquad \mathrm{N}(\%)\end{array}$ \\
\hline \multicolumn{3}{|c|}{ Características sociodemográficas } \\
\hline \multicolumn{3}{|c|}{ Sexo } \\
\hline Feminino & $10(76,92)$ & $9(90,00)$ \\
\hline Masculino & $3(23,08)$ & $2(66,67)$ \\
\hline \multicolumn{3}{|l|}{ Idade } \\
\hline $20-30$ & $3(23,08)$ & $2(66,67)$ \\
\hline $31-40$ & $3(23,08)$ & $2(66,67)$ \\
\hline $41-60$ & $5(38,46)$ & $5(100)$ \\
\hline $61-70$ & $2(15,38)$ & $2(100)$ \\
\hline \multicolumn{3}{|l|}{ Cor } \\
\hline Pardo & $10(76,92)$ & $8(80,00)$ \\
\hline Branco & $0(0)$ & $0(0)$ \\
\hline Negro & $3(23,08)$ & $3(100)$ \\
\hline \multicolumn{3}{|l|}{ Escolaridade } \\
\hline Médio incompleto & $2(15,38)$ & $2(100)$ \\
\hline Médio completo & $8(61,55)$ & $7(87,50)$ \\
\hline Superior incompleto & $1(7,69)$ & $0(0)$ \\
\hline Superior completo & $2(15,38)$ & $2(100)$ \\
\hline \multicolumn{3}{|l|}{ Renda } \\
\hline Até 1 salário & $6(46,15)$ & $5(83,33)$ \\
\hline 1-2 salários & $6(46,15)$ & $6(100)$ \\
\hline 2-3 salários & $1(7,70)$ & $0(0)$ \\
\hline \multicolumn{3}{|c|}{ Características ambientais } \\
\hline \multicolumn{3}{|c|}{ Material da casa } \\
\hline Alvenaria & $12(92,30)$ & $10(83,33)$ \\
\hline Madeira & $1(7,70)$ & $1(100)$ \\
\hline \multicolumn{3}{|c|}{ Escoamento de esgoto } \\
\hline Rede geral & $2(15,38)$ & $1(50,00)$ \\
\hline Fossa & $10(76,92)$ & $9(90,00)$ \\
\hline Vala/rio & $1(7,70)$ & $1(100)$ \\
\hline \multicolumn{3}{|c|}{ Saúde e hábitos de higiene } \\
\hline \multicolumn{3}{|c|}{ Tratamento da água } \\
\hline Filtro de barro & $7(53,84)$ & $7(100)$ \\
\hline Fervura e cloro & $1(7,70)$ & $0(0)$ \\
\hline Mineral & $3(23,08)$ & $3(100)$ \\
\hline Filtro com vela & $2(15,38)$ & $1(50,00)$ \\
\hline
\end{tabular}




$\begin{array}{lcc}\begin{array}{l}\text { Lavagem das mãos após usar banheiro } \\ \text { Sempre }\end{array} & 10(76,92) & 8(80,00) \\ \begin{array}{l}\text { Nunca } \\ \text { Lavagem das mãos após contato com } \\ \text { animais }\end{array} & 3(23,08) & 3(100) \\ \begin{array}{l}\text { Sempre } \\ \text { Às vezes }\end{array} & & 8(88,89) \\ \text { Lavagem das mãos antes de alimentar-se } & 9(69,23) & 3(75,00) \\ \text { Sempre } & 4(30,77) & 8(80,00) \\ \text { Às vezes } & 10(76,92) & 3(100) \\ \text { Tratamento das frutas e verduras } & 3(23,08) & 8(100) \\ \text { Com água } & & 0(0) \\ \text { Água e sabão } & 8(61,54) & 3(100) \\ \text { Água sanitária } & 2(15,38) & \\ \text { Tratamento do leite } & 3(23,08) & 3(100) \\ \text { Fervura } & & 8(80,00) \\ \text { Nenhum } & 3(23,08) & \\ \text { Realizou exames fecais } & 10(76,92) & 9(90) \\ \text { Sim } & & 2(66,66) \\ \text { Não } & 10(76,92) & 1(50) \\ \text { Quando realizou os exames } & 3(23,08) & 7(87,5) \\ \text { Recente } & & \end{array}$

Fonte: Autores.

\section{Discussões}

Todos os participantes deste trabalho receberam o resultado do exame parasitológico. Aqueles com diagnóstico positivo para endoparasitas foram orientados a procurar as unidades de saúde do município para tratamento específico.

A prevalência endoparasitária encontrada nos idosos das duas instituições analisadas foi de 57,98\%, maior do que em outros estudos que também analisaram idosos institucionalizados no Brasil, que apresentaram taxas de 36,48\% em Minas Gerais (Valadão et al., 2017), 30,65\% no Rio de Janeiro (Macharetti et al., 2014), 30,5\% na Bahia (Santos et al., 2017) e 6,54\% em Santa Catarina (Quadros et al., 2018). As infecções endoparasitárias, mesmo quando moderadas, tornam-se preocupantes em hospedeiros imunodeprimidos ou com diminuição das funções normais do sistema imune, como os idosos, portanto é essencial que eles sejam examinados para se detectar a presença de parasitas intestinais através de exames periódicos. Os idosos mais vulneráveis sofrem não só os efeitos sobre o seu estado imunológico, como também as repercussões sobre o seu estado nutricional (Albright \& Albright, 1994; Wu \& Meydani, 2008).

Embora as duas instituições analisadas apresentaram prevalências expressivas, Centro de Convivência 46,98\%\% e ILP 83,33\%, a prevalência endoparasitária nos idosos da Instituição de Longa Permanência foi significativamente mais alta. Os funcionários que trabalham na ILP também apresentaram uma alta taxa de prevalência. O cálculo da razão de probabilidade (Odds Ratio) para infecções endoparasitárias nos idosos da Instituição de Longa Permanência comparada com os idosos do Centro de Convivência, mostrou que os idosos da ILP apresentaram aproximadamente 7 vezes maior chance de se infectar com parasitas intestinais quando comparado aos idosos do Centro de Convivência. Quando calculado o OR para infecções endoparasitárias nos idosos da Instituição de Longa Permanência em comparação com os funcionários da mesma instituição, os idosos apresentaram aproximadamente 1,8 vezes maior chance de se infectar quando comparados com os funcionários. Porém o valor de $p$ apresentado nessa variável é maior que 0,005 , indicando que não existe uma diferença estatisticamente significante entre esses dois grupos.

Estes resultados sugerem uma condição epidemiológica preocupante da população idosa da ILP pois os altos índices de prevalência tanto nos idosos quanto nos funcionários evidenciam o alto risco de infecção e reinfecção nesse ambiente. 
Muitos idosos residentes na ILP estão em estado de demência e, portanto, são mais vulneráveis às infecções, o que pode explicar as taxas elevadas. A perda progressiva da autonomia para o autocuidado com frequente prejuízo da higiene pessoal e dos alimentos estando totalmente dependentes de cuidadores é um fator determinante na infecção parasitária (Richards, 2002; Amós \& Hogla, 2005).

Os resultados obtidos mostraram um predomínio de protozoários nas amostras tanto dos idosos das duas instituições como dos funcionários da ILP. Os endoparasitas patogênicos Criptosporidium sp., Cyclospora sp. e Giardia lamblia foram os mais prevalentes, com 22\%, 19\% e 18\%, respectivamente. Esses protozoários intestinais são encontrados na água e nos alimentos e estão associados a hábitos de higiene pouco confiáveis (Neves et al., 2016). Eles são de grande importância na saúde pública devido os graves quadros de diarreia agudam ou crônicas, gastroenterites prolongadas e máabsorção intestinal que podem causar (Rey, 2008; Barros, 2015). A prevalência desses protozoários está relacionada a aglomerados humanos, portanto, as Instituições de Convivência e de Longa Permanência de idosos tornam-se locais propícios. (Carvalho, 2009).

A prevalência de endoparasitas comensais como Entamoeba coli, Endolimax nana e Blastocystis hominis, embora menos expressivas nas amostras analisadas, são importantes por servir de indicadores de condições sanitárias precárias além de contaminação ambiental e habitacional que indica situação de risco de infecção por outros agentes patogênicos que possuem o mesmo tipo de contágio, fecal-oral. (Menezes et al., 2013).

A maioria das infecções observadas neste estudo foram co-infecções, sendo 38,46\% das amostras positivas dos idosos do Centro de Convivência, 76,66\% dos idosos da ILP e 69,23\% dos funcionários da ILP. As co-infecções mais observadas foram as associações duplas de Cryptosporidium sp. + Cyclospora sp. e associações triplas de Giardia lamblia + Cryptosporidium sp. + Cyclospora sp. O poliparasitismo observado nos três grupos analisados neste estudo, idosos e funcionários, pode ser explicado por fatores de risco compartilhados para infecções endoparasitárias, como maus hábitos de higiene e o fato de que os idosos (especialmente da ILP) são dependentes dos funcionários cuidadores e não recebem tratamento adequado para as parasitoses intestinais.

É importante destacar que quando os parasitas acometem isoladamente os idosos, geralmente não apresentam alta letalidade, porém as co-infecções com parasitas patogênicos como observado neste estudo, afetam o estado nutricional do idoso, podendo causar sangramento intestinal e outras complicações graves (Macharetti et al, 2014; Barros, 2015).

Esta pesquisa mostrou que a prevalência de parasitas intestinais entre homens e mulheres foi semelhante tanto nos idosos do ILP quanto nos idosos do Centro de Convivência. Esse resultado sugere que as infecções não dependem do sexo, conforme observado também em outros estudos (De Carli et al., 1997; Ishiyama et al., 2003; Neves, 2016). Quanto a faixa etária a pesquisa identificou a maior frequência de idosos parasitados na faixa de 60 a 70 anos, e a menor esteve nos idosos $\geq$ 81 anos, provavelmente pelo fato de que nesse grupo de maiores de 81 anos muitos estão em estado de demência e tem menos contanto com o ambiente externo. Esses achados corroboram o estudo de Hurtado-Guerreiro et al. (2005), o qual a maior prevalência de endoparasitas foi registrada na faixa de 60 a 69 anos e a menor esteve na faixa de 80 a 94 anos.

A contaminação por parasitas intestinais ocorre, na maioria dos casos, por via oral, através da ingestão de água ou alimentos contaminados. A ausência ou insuficiência nas condições sanitárias e práticas inadequadas de higiene pessoal são importantes mecanismos de transmissão (Prado et al., 2001). Portanto, a fim de verificar a qualidade sanitária e ambiental das duas instituições, justificada pela alta prevalência de idosos infectados, e avaliar os pontos de fragilidades, foram realizadas avaliações nos espaços institucionais. Assim pontos relevantes foram observados nas duas instituições como, o destino final do esgoto é a fossa séptica, a água é encanada da rede pública e também obtida de poço artesiano e a purificação da água para consumo é feita por filtros. De acordo com Neves (2016), alguns protozoários patogênicos como a G. lamblia e 
Cryptosporidium sp., os mais prevalentes nas duas instituições, são transmitidos pela água consumida e podem resistir até a ação do cloro adicionado à água tratada.

O déficit de esgotamento sanitário no Município de Cáceres - MT, vem gerando como consequência o aumento de construção de sistema individual principalmente fossas rudimentares. Essa alternativa de destinação final é uma opção de 92,8\% da população do município de Cáceres (Trata-Brasil,2018), por ser mais barata, porém, é a menos segura, trazendo impactos sobre o ambiente e sobre a saúde dos moradores, sendo uma das principais fontes de contaminação do solo e da água com efeitos nocivos à saúde devido a presença de microrganismo patogênicos (PMSB, 2015).

As instituições abrigam animais domésticos não vermifugados como gatos, cães e aves, e nos espaços há hortas que utilizam esterco animal para o cultivo de hortaliças que são consumidas pelos idosos. Esses fatores são extremamente relevantes já que muitos animais, através de suas fezes, constituem uma significativa fonte de infecção parasitária (Rosales, Malheiros, 2017; Zanetti et al., 2019; Zanetti et al., 2021a; 2021b), e podem ser os responsáveis pela contaminação do ambiente e (re) infecção dos idosos.

Os coordenadores das duas instituições relataram que nenhuma palestra de educação e saúde voltada para os idosos foram realizadas, e que os funcionários que atuam diretamente com os idosos, não possuem treinamento adequado em relação a higiene pessoal e o uso dos equipamentos de proteção individual. As altas prevalências de parasitas intestinais nos idosos, principalmente na ILP, bem como nos funcionários da ILP, demonstram que o convívio dos profissionais com os idosos é um fator de exposição às parasitoses, pois, a transmissão direta de pessoa para pessoa ocorre principalmente em comunidades fechadas como as Instituições de Longa Permanência devido o contato constante entre idosos, enfermeiros e manipuladores de alimentos (Thompson, 2000; Girotto, 2013), e que muitas vezes, como observado nessa pesquisa, não são treinados quanto aos procedimentos corretos de higiene.

A promoção de educação em saúde é um fator crucial para o desenvolvimento de ações de combate e prevenção contra parasitas intestinais, pois o desconhecimento a respeito das medidas preventivas é condicionante para a disseminação das endoparasitoses (Camello et al., 2016). E conforme os coordenadores das duas instituições relataram, nenhuma palestra ou atividade educativa é realizada nesses ambientes, evidenciando a falha das ações e programas sociais/educacionais da região.

Esse estudo avaliou ainda as características sociodemográficas e hábitos de higiene pessoal dos idosos do Centro de Convivência e dos funcionários da ILP. Não foi possível aplicar o questionário com os idosos da ILP, pois a maioria possuí algum tipo de demência. Foi realizado o cálculo da razão de probabilidade - Odds Ratio (OR) para todas as variáveis sociodemográficas e hábitos de higiene pessoal dos idosos e dos funcionários, porém nenhuma apresentou significância estatística. Embora sem significância estatística das prevalências parasitárias com nenhuma das variáveis analisadas, vale destacar alguns pontos.

Em relação à renda, a maioria dos idosos, assim como dos funcionários, recebem de um a dois salários mínimos, e muitos idosos dependem da ajuda de programas sociais. O grau de instrução também é baixo entre os idosos, a grande maioria não completou o ensino fundamental ou são analfabetos. Entre os funcionários a maioria possuí o ensino médio completo. O baixo nível socioeconômico e pouca educação afeta diretamente a situação de saúde da população, cujos padrões de vida, de higiene ambiental e de educação sanitária deficientes, constituem os principais fatores de risco para o acometimento de endoparasitos (Borges et al., 2011).

Hábitos de higiene como lavagem das mãos antes da refeição e depois de usar o banheiro foram relatados pelos idosos e funcionários entrevistados, embora muitos deles afirmaram que nem sempre tomam esses cuidados. $\mathrm{O}$ ato de lavar as mãos é muito importante para redução de riscos de contaminação por endoparasitas, reafirmando que a educação sanitária é uma importante medida que deve ser implantada no combate aos patógenos (Larré et al., 2014). 
Tanto os idosos quanto os funcionários analisados nesta pesquisa, relataram a realização de exames parasitológicos em algum momento, porém mencionaram a realização a mais de um ano. Isto demonstra a ausência da atenção primária de saúde para realização de exames de rotina e tratamento antiparasitário nesse público de idosos institucionalizados e funcionários que aí atuam. As Estratégias de Saúde da Família devem ser mais participativas e atuantes para levar a essas instituições, práticas de prevenção e ações de saúde que diminuam a incidência das parasitoses.

Este estudo apresenta como limitações o fato de ser um inquérito local da situação dos idosos dessas duas instituições existentes na cidade. Uma investigação em outras instituições poderia abordar um cenário mais amplo da situação dos idosos institucionalizados no Brasil. Ainda como limitações existe o fato de ter sido coletado apenas uma amostra fecal de cada idoso, pois em alguns casos a positividade depende de estágios detectáveis dos parasitas, o que pode ter subestimado a prevalência das parasitoses intestinais verificada neste estudo.

\section{Conclusão}

Os resultados chamam a nossa atenção para as condições sanitárias e de saúde da população idosa que vive em instituições na cidade de Cáceres-MT. Altas prevalências de parasitas intestinais foram identificadas entre os idosos do Centro de Convivência (46,98\%), idosos da Instituição de Longa Permanência (83,33\%) e funcionários $(84,61 \%)$. Os resultados mostram uma condição epidemiológica preocupante da população idosa da ILP, pois os altos índices de prevalência tanto nos idosos quanto nos funcionários evidenciam o alto risco de infecção e reinfecção nesse ambiente. Os parasitas patogênicos Cryptosporidium sp., Ciclospora sp. e Giardia lamblia foram os mais prevalentes com altas taxas observadas. Embora dados importantes foram obtidos dos grupos analisados, não foi verificada associação estatística significativa entre as prevalências parasitárias e características socioambientais, econômicas e hábitos de higiene dessa população. É notável a necessidade de programas educacionais de conscientização das práticas higiênicas e de atenção básica a saúde da população idosa nessas instituições. Portanto é necessário um posicionamento e ações da sociedade, bem como do poder público, voltado para melhoria da qualidade de vida da população idosa.

Os resultados deste estudo fornecem informações úteis para a concepção de estratégias de prevenção de infecções parasitárias e intervenções adequadas em idosos das regiões do pantanal Brasileiro a fim de melhorar suas condições de vida.

\section{Agradecimentos}

Os autores agradecem a diretoria, funcionários e idosos da ILP Servas de Maria e do Centro de Convivência Remanso Fraterno pelo aceite e empenho na participação dessa pesquisa. Agradecem ainda, o apoio financeiro do Conselho Nacional de Desenvolvimento Científico e Tecnológico - CNPq (Projeto Universal 423391/2018-6).

\section{Referências}

Albright, J. W., \& Albright J. F. (1994) Ageing alters the competence of the immune system to control parasite infection. Immunol Lett..

Barros, S. V. A. (2015) Contribuição para o estudo da criptosporidiose em vitelos de explorações leiteiras da ilha terceira, Açores. 2015. 96p. Dissertação (Medicina Veterinária) - Universidade de Lisboa, Lisboa.

Borges, W. F., Marciano, F. M. \& Oliveira, H. B. (2011). Parasitos intestinais: elevada prevalência de Giardia lamblia em pacientes atendidos pelo serviço público de saúde na região sudeste de Goiás, Brasil. Revista de Patologia Tropical, 40(2), 149 - 157.

Camello J. T, et al. (2016). Prevalência de parasitoses intestinais e condições de saneamento básico das moradias em escolares da zona urbana de Caxias do Sul, Rio Grande do Sul. Scientia Medica, 26(1), 1-6.

Carvalho T. T. R. (2009). Estado atual do conhecimento de Cryptosporidium e Giardia.Revista Patologia Tropical, $38:$ 1-16.

Costa, P. S. C. B. V.et al. (2014). Prevalência de anemia e enteroparasitoses em um laboratório da periferia da cidade de Parnaíba-Pi. R. Interd, 7(3), 71-76. 
De Carli, G. A., et al., (1997). Prevalência das enteroparasitoses na população urbana e rural da região carbonífera da cidade de Arroio dos Ratos, no Estado do Rio Grande do Sul. Revista Brasileira de Análises Clínicas, 78(4): 83-5.

Ely, L. S. et al., (2011). Prevalência de enteroparasitos em idosos. Revista Brasileira de Geriatria e Gerontologia, 14(4), 637-646.

Freitas, E. V. et al., (2016). Tratado de geriatria e gerontologia. (4a ed.), Guanabara Koogan.

Girotto, K. G. et al., (2013). Prevalence and risk factors for intestinal protozoa infection in elderly residents at Long Term Residency Institutions in Southeastern Brazil. Revista do Instituto de Medicina Tropical de São Paulo, 55(1), 19-24.

Hurtado-Guerrero, A. F., Alencar, F \& H., Hurtado-Guerrero, J. C. (2005). Ocorrência de enteroparasitas na população geronte de Nova Olinda do Norte Amazonas, Brasil. Acta Amazonica, 35(4), 487-490.

IBGE, Instituto Brasileiro de Geografia e Estatística. Síntese de indicadores sociais, uma análise das condições de vida da população brasileira. 2012.

Ishiyama, S., et al., (2003). A small scale study on intestinal parasitosis in a remote village in Nepal. Nepal Medical College Journal, 5: 28-30.

Justino, G. D. O et al. (2010). Identificação e tratamento de endoparasitoses em pacientes da terceira idade. p. 6.

Larré, A. B., \& Alegre, P. (2014). Pontifícia universidade católica do Rio Grande do Sul instituto de geriatria e gerontologia programa de pós-graduação em gerontologia biomédica. p. 14.

Macharetti, H., Norberg, A. N. \& Martins, J. S. A. (2014). Protozoários e helmintos em interação com idosos albergados em lares geriátricos no estado do Rio de Janeiro, brasil. p. 10.

Menezes, R. A. D. et al. (2013). Enteroparasitoses em pacientes atendidos no laboratório de uma unidade de saúde de Macapá, Amapá, 2011 - 2012. Revista de Biologia e Ciências da Terra, 13(1), 191 - 198

Neves, D. (2016). Neves - Parasitologia Humana, (13a ed.), Edição Atheneu.

Prado, M. S., Barreto, M. L., Strina, A., Faria, J. A. S., Nobre, A. A., \& Jesus, S. R. (2001). Prevalência e intensidade da infecção por parasi- tas intestinais em crianças na idade escolar na cidade de Sal- vador (Bahia, Brasil). Rev Soc Bras Med Trop, 34(1):99-101.

Quadros, R. M. \& Miguel, R. L. (2018). Cryptosporidium spp. Em idosos residentes em instituições de longa permanência no sul do Brasil. Clinical \& Biomedical Research, 38(3), 223-228.

Rey, L. (2008). Parasitologia: parasitos e doenças parasitárias do homem nos trópicos ocidentais. Guanabara Koogan.

Rouquayrol M. Z, \& Filho N. A., (2003). Epidemiologia \& Saúde. Guanabara Koogan, 736p.

Rosales, T. F. L., \& Malheiros, A. F. (2017). Contaminação Ambiental por enteroparasitas presentes em fezes de cães em uma região do Pantanal. $O$ Mundo da Saúde, 41(3), 368-377.

Saito, R. K. A 2012). Importância da análise do solo nos estudos epidemiológicos sobre parasitoses intestinais. p. 6.

Santos, P. H. S. et al. (2017). Prevalence of intestinal parasitosis and associated factors among the elderly. Revista Brasileira de Geriatria e Gerontologia, 20(2), 244-253

Thompson, R. C. A. (2000). Giardíase como doença infecciosa reemergente e seu potencial zoonótico. Int J Parasitol., 30: $1259-67$.

Trata-Brasil (2018). Esgotamento Sanitário Inadequado e Impactos na Saúde da População. Estudo: Impactos na Saúde e no Sistema Único de Saúde Decorrentes de Agravos Relacionados ao Esgotamento Sanitário Inadequado dos 100 Maiores Municípios no Período 2008 -2011.

Valadão, A. F., et al., (2017). Parasitoses intestinais em idosos institucionalizados no município de Ipatinga - Minas Gerais. Brazilian Journal of Surgery and Clinical Research-BJSCR. 18(1), 10-17.

Wu, D. \& Meydani, S. N. (2008). Age-associated changes in immune and inflammatory responses. J. Leukocyte Biol, 84: 1-15.

Zanetti, A. S, Silva B. L. Z, Barros, L. F, Garcia, H. A, Aguiar, D. M, Espinosa, A. O., \& Malheiros, A. F. (2021). Investigação epidemiológica de parasitas gastrointestinais em cães de diferentes ambientes da região central do Brasil: implicações para a saúde humana-animal-ambiental. Research, Society and Development, 10(1) e31210111751

Zanetti, A. S, Barros, L. F, Araújo, M. S. M, Garcia, H. A, Aguiar, D. M, Espinosa, A. O., \& Malheiros, A. F. (2021). Diversity and prevalence of intestinal parasites of zoonotic potential in animal hosts from diferente biomes in the central region of Brazil. Annals of Parasitology. 66 (1), 91-101. 